\title{
Assessment of adhesion formation after laparoscopic intraperitoneal implantation of Dynamesh IPOM mesh
}

Andrzej Jamry ${ }^{1}$, Marek Jałyński², Łukasz Piskorz ${ }^{3}$, Marian Brocki ${ }^{3}$

1District Hospital, Starachowice, Poland

2Surgery and Roentgenology Chair, Warmian-Masurian University, Olsztyn, Poland

${ }^{3}$ Department of Chest, General and Oncological Surgery, Medical University of Lodz, Poland

Submitted: 29 June 2011

Accepted: 26 September 2011

Arch Med Sci 2013; 9, 3: 487-492

DOI: $10.5114 /$ aoms.2013.35345

Copyright $\odot 2013$ Termedia \& Banach

\begin{abstract}
Introduction: Formation of adhesions after laparoscopic hernia repair using the intra-peritoneal onlay mesh (IPOM) procedure can lead to intestinal obstruction or mesh erosion into intestinal lumen. The aims of this study included: measurement of adhesion formation with Dynamesh IPOM after laparoscopic intraperitoneal implantation, and assessment of the occurrence of isolated adhesions at the fastening sites of slowly absorbable sutures.

Material and methods: Twelve healthy pigs underwent laparoscopic implantation of 2 Dynamesh IPOM mesh fragments each, one was fastened with PDSII, and the other with Maxon sutures. An assessment of adhesion formation was carried out after 6 weeks and included an evaluation of surface area, hardness according to the Zhulke scale, and index values. The occurrence of isolated adhesions at slowly absorbable suture fixation points was also analyzed.

Results: Adhesions were noted in $83.3 \%$ of Dynamesh IPOM meshes. Adhesions covered on average $37.7 \%$ of the mesh surface with mean hardness 1.46 and index value 78.8. In groups fixed with PDS in comparison to Maxon sutures adhesions covered mean $31.6 \%$ vs. $42.5 \%(p=0.62)$ of the mesh surface, mean hardness was 1.67 vs.1.25 ( $p=0.34)$ and index 85.42 vs. $72.02(p=0.95)$.

Conclusions: The Dynamesh IPOM mesh, in spite of its anti-adhesive layer of PVDF, does not prevent the formation of adhesions. Adhesion hardness, surface area, and index values of the Dynamesh IPOM mesh are close to the mean values of these parameters for other commercially available 2-layer meshes. Slowly absorbable sutures used for fastening did not increase the risk of adhesion formation.
\end{abstract}

Key words: adhesions, surgical meshes, laparoscopy.

\section{Introduction}

Intra-abdominal adhesions occur after more than half of all abdominal operations and are a major cause of post-operative complications. They attach normally separated organs to each other and cause small bowel obstruction, chronic pelvic pain, dyspareunia, infertility, and higher complication rates in subsequent operations [1].

Patients with hernias who undergo incisional hernia repair with polypropylene meshes (PPM) placed in their peritoneal cavity experience adhesions on up to $80-100 \%$ of the mesh surface, along with an increased risk of acute inflammatory responses, recurrences, enteric fistula and mesh migration [2-9].
Corresponding author: Andrzej Jamry MD, PhD II Surgical Departament District Hospital

Starachowice, Poland

Phone: +48 602795259

E-mail: jamry@tlen.pl 
In order to minimize the frequency and severity of adhesions, mesh grafts with anti-adhesive properties relative to the viscera have been introduced. These have included meshes with coated implants [9] and 2-layered grafts whose parietal surface enables proper anchoring while their visceral surface prevents adhesions between the primary layer and bowel [10,11]. The external layer of these meshes is often built of polypropylene or polyester, while the inner layer is made of collagen (Parietex), cellulose (Proceed), expanded polytetrafluoroethylene (ePTFE, dual-mesh) or polyglactin 910. Even though these materials were designed specifically to prevent adhesions, in actual practice they adhere to viscera on $18-57 \%$ of their surface [12-16].

To reduce adhesion formation and other adverse consequences, implants made of newer materials and with modified construction such as different pore sizes, surface area and filament structure are constantly being tested [17-20]. One of these is the Dynamesh IPOM mesh, whose visceral surface is made of a polymer, polyvinylidene fluoride (PVDF) [21]. The PVDF meshes are characterized by improved biostability, decreased bending stiffness, and a lowered tissue response relative to those made of polypropylene (PP), polyethylene-terephthalate or polytetrafluoroethylene. They are also cheaper relative to the most commonly used 2-layer Dual mesh.

Fixing elements can be an additional, independent cause of visceral adhesion after hernia surgery involving the use of a synthetic mesh. For example, while mesh anchorage is generally more successful with ProTack or AbsorbaTack relative to the I-clip, ProTack staples can cause isolated adhesions

Table I. Classification of IPOM meshes according to percent surface area covered by adhesions at 6 weeks after surgery

\begin{tabular}{|lcc|}
\hline Groups & $\begin{array}{c}\text { Surface area } \\
\text { covered [\%] }\end{array}$ & $\begin{array}{c}\text { Mesh } \\
\text { distribution [\%] }\end{array}$ \\
\hline 1 & 0 & 16.7 \\
\hline 2 & $1-25$ & 37.5 \\
\hline 3 & $26-50$ & 12.5 \\
\hline 4 & $51-75$ & 12.5 \\
\hline 5 & $76-100$ & 20.8 \\
\hline
\end{tabular}

Table II. Classification of IPOM meshes according to adhesion hardness at 6 weeks after surgery

\begin{tabular}{|lcc|}
\hline Groups & $\begin{array}{c}\text { Zhulke scale } \\
\text { of hardness }\end{array}$ & $\begin{array}{c}\text { Mesh } \\
\text { distribution [\%] }\end{array}$ \\
\hline 1 & 0 & 16.7 \\
\hline 2 & 1 & 37.5 \\
\hline 3 & 2 & 33.3 \\
\hline 4 & 3 & 8.3 \\
\hline 5 & 4 & 4.2 \\
\hline
\end{tabular}

due to their parts jutting over the prosthesis [21]. This problem can be solved by the use of long-lasting, slowly absorbable monofilament threads such as PDSII and Maxon sutures, used in the present study for mesh stabilization.

The aims of our study were to measure the frequency, surface area, and hardness of adhesions formed after laparoscopic intra-peritoneal implantation of the Dynamesh IPOM mesh, and to assess whether isolated adhesions are formed in response to slowly absorbable sutures used for prosthesis fastening.

\section{Material and methods}

Twelve healthy swine of the Polish Large White (PLW) breed without hernias or incisions, aged 12 weeks and weighing between $30 \mathrm{~kg}$ and $40 \mathrm{~kg}$ were included in this study. All operations were performed by the same team (JA, JM) using the same surgical technique.

For each laparoscopy, a $10 \mathrm{~mm}$ optical trocar, $5 \mathrm{~mm}$ working trocar and a device of a "passer suture" type were used to implant 2 fragments of Dynamesh IPOM mesh of $6.0 \mathrm{~cm} \times 6.6 \mathrm{~cm}$. Fastening was carried out with the aid of 4 slowly absorbable sutures through all the layers, inserted in subcutaneous tissue and placed $4 \mathrm{~cm}$ apart from each other.

Within each animal, one mesh fragment was stabilized using absorbable PDSII sutures (Ethicon), while the other was fixed with Maxon sutures (Covidien). The post-surgical period of observation lasted for 6 weeks.

Initial observations included an analysis of the mesh surface occupied by adhesions, expressed as a percentage of their surface area. Adhesion hardness was classified using the Zhulke scale, in which 0 = lack of adhesions, 1 = delicate adhesions separated "at pace", 2 = stronger adhesions, separated partly to sharply plus single vessels, 3 = strong adhesions separated sharply, and $4=$ very strong as in 3 plus very strongly adhered organs so that an attempt to separate them can lead to their damage [6].

Additionally, due to considerable divergence observed between the surface area and adhesion hardness, an evaluation of the association between these 2 features was carried out. Meshes were classified into 5 groups according to adhesion surface area and the percentage share of each of them was calculated (Table I). Next, adhesion hardness was evaluated according to the Zhulke scale in the same groups (Table II). This allowed for a comparison of both sets of values within and between groups (Figure 1).

Based upon these data, the conformity of both parameters occurs only in groups 1 and 2, which contained adhesions of the smallest surface and 


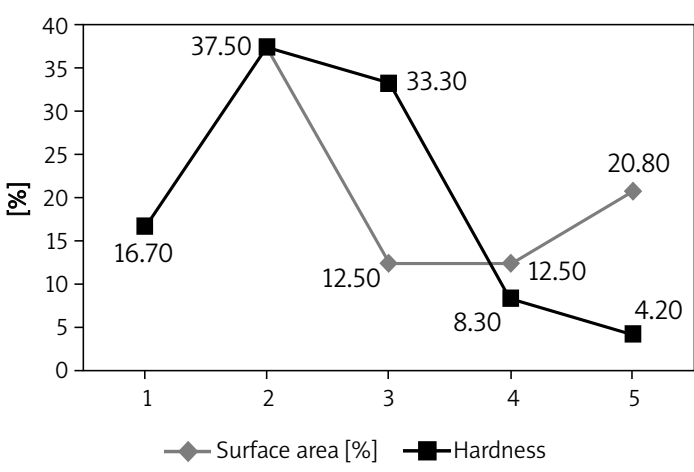

Figure 1. Relationship between percent surface area and adhesion hardness within groups of IPOM meshes

with the least hardness. On the other hand, in groups 3, 4, and 5 considerable divergences were observed between them.

These findings support our hypothesis that an adhesion index (incorporating both adhesion surface area expressed as a percentage and adhesion hardness) should be used for a proper and complete evaluation of adhesion formation after laparoscopic intra-peritoneal implantation [6].

The second aim of this study was to observe whether isolated adhesions are formed at the points of anchoring of the slowly absorbable sutures. Each of the 96 points of fixation was evaluated in this way. Additionally, mean values of surface area, hardness, and index for the group of meshes fixed with PDSII and Maxon sutures were calculated and compared to determine whether the type of suture used had an influence on adhesion formation.

These studies were performed with the permission of the Ethics Commission of Warmian-Masurian University (Resolution No. 02/2009).

\section{Results}

Analysis of the data revealed adhesions in 20/24 implanted meshes (83.3\%). The mean value of the adhesion surface area was $37.7 \%$, while mean hardness was 1.46 according to the Zhulke scale, and the mean index value was 78.75 .

The distribution of adhesion index values together with the type of suture used in individual swine demonstrates substantial divergence. In meshes fixed with PDS, as compared to Maxon, adhesions covered on average $32.5 \%$ vs. $42.5 \%(p=0.62)$ mesh surface, with mean hardness 1.67 vs. 1.25 $(p=0.34)$ and the index of 85.45 vs. 72.08 ( $p=0.95)$ (Figure 2). Since the same operational technique was used on all animals by the same team, these data indicate the strong influence of individual susceptibility in adhesion formation.

Based on previous studies and direct experience in clinical practice, infection is known to cause hard adhesion around inflammatory foci. Mesh infections

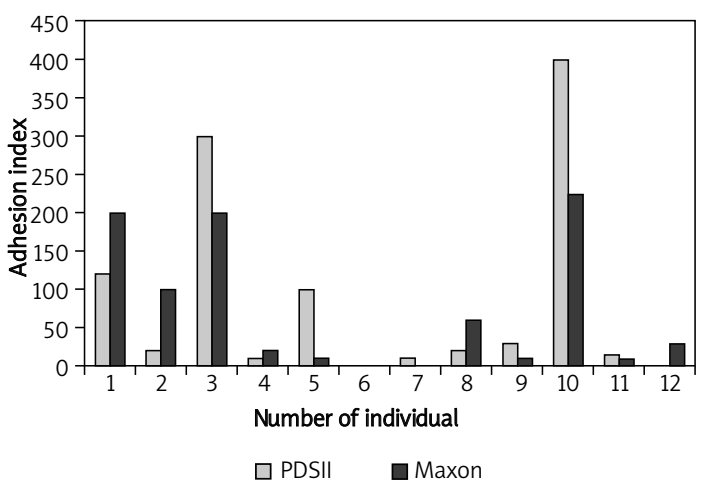

Figure 2. Adhesion index values within individual swine

usually require surgery. Interestingly, in the present study, despite infections in the suture canals, the meshes themselves were not infected.

Specifically, infections were observed in 3 instances around sutures. In individual 1, the mesh fastened with PDSII had an adhesion covering 60\% of its surface, while the mesh fastened with Maxon had an infection around it and was covered by an adhesion over $100 \%$ of its surface. However, separation of viscera from both meshes was relatively easy - grade 2 according to the Zhulke classification - giving a total index of 120 for the PDSII and 200 for the Maxon sutures.

In individual 6, in spite of an infection around the Maxon suture fastening the Dynamesh, no adhesions were observed on either mesh. In individual 8 , adhesions were present over $10 \%$ of the surface of the mesh fastened with PDSII with a hardness of level 2 (index 20), and over $60 \%$ of the surface of the mesh fastened with Maxon with a hardness of level 1 (index 60). These values were significantly lower relative to those calculated for PDSII meshes in individuals 3 (index 300) and 10 (index 400).

Clearly, in our study infections had no influence on adhesion occurrence or hardness, which could not be distinguished from adhesions on meshes without infections. This finding is surprising and requires further observation and analysis.

In the second part of the present study, no isolated adhesions were observed at any of the 96 fastening points for the slowly absorbable sutures. Additionally, no significant differences were observed in surface area, adhesion hardness or index for meshes fixed with either PDSII or Maxon sutures (Figure 3).

\section{Discussion}

Intra-abdominal adhesions cause many postoperative complications in patients who undergo incisional hernia repair. These patients may experience adhesions on up to $80-100 \%$ of the mesh surface, along with an increased risk of other adverse con- 


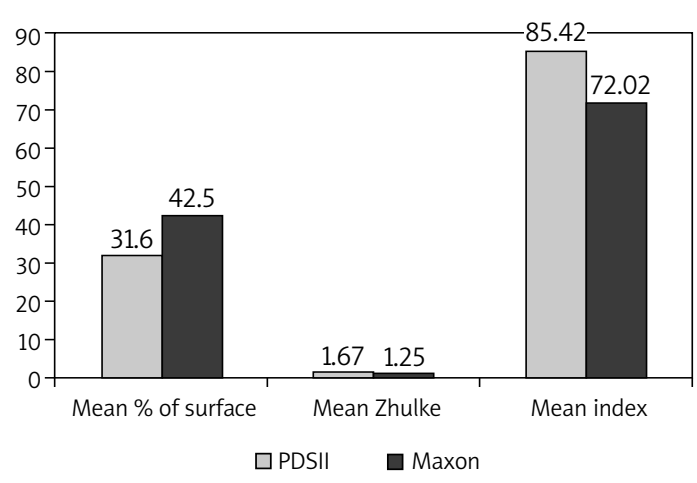

Figure 3. Comparison of the mean surface area, adhesion hardness (Zhulke scale) and index for meshes fixed with PDSII relative to Maxon sutures

sequences such as small bowel obstruction, chronic pelvic pain, infertility, fistula, mesh migration, and higher complication rates in subsequent operations [2-9]. Additionally, nearly one-third of these patients experience recurrences and may require re-hospitalization and further surgery [22].

The influence of the laparoscopic technique and the effects of introducing a foreign body such as a mesh into the peritoneal cavity are still the subject of debate and study [23]. Our knowledge on the subject of adhesions forming after mesh implantation cannot, for obvious reasons, be based on direct assessment in patients. Indirect information obtained on the basis of analysis of viscera migrations and post-operative adhesions to the abdominal wall using ultrasonography (USG) or MRI examinations are imprecise and unsuitable for interpretation $[1,24]$. As a result, the literature does not contain an official definition of adhesions or an objective system of classification of their extent and severity. There are also no standard clinical guidelines for their diagnosis and treatment.

This inability to accurately measure the extent of mesh surface area covered by adhesions and properly evaluate adhesion hardness influences risk assessment of adhesion formation. For example, viscera that adhere to a small surface and require force to separate can constitute a bigger risk of complications than relatively softer adhesions over a larger surface area.

At the same time, there is no unambiguous correlation between hardness and the extent of adhesion formation, as confirmed by the present study. Taking into account both parameters to calculate their index makes it possible to prioritize the data in terms of the complication risk $[14,15]$. Thanks to the increasingly accurate sources of information based on experimental studies, both parameters can now be evaluated [24].

On the other hand, results obtained in the laboratory setting may not accurately predict adhesion formation in humans. Each species may respond differently to implanted materials and parietal and visceral damage on separation of adhesions from the hernia sac, limiting the application of these findings to human clinical practice $[25,26]$.

Variations in laboratory animal species, size, in the structure and type of materials used to synthesize prostheses, surgical techniques and criteria of evaluation can all influence the experimental consequences including inflammatory reactions, calcification, infection risk and finally adhesion formation [1, 13, 18, 23, 27].

Clinically, mesh implants are intended to be used over the entire duration of a lifetime (50-70 years). Therefore, experimental observations made during a relatively limited time period are not very useful in clinical practice. Similarly, while longer clinical observations are available for polypropylene meshes, newer meshes have a much shorter history of usage and information on their quality and durability is limited $[2,16,20,28]$. Therefore the relationship between adhesion formation and mesh type used requires further, systematic analysis.

Polypropylene is widely used in surgery. Because of its association with formation of enterocutaneous fistulae and adhesions, the adhesive potential of modified polypropylene meshes and their usefulness in laparoscopic ventral hernia repair (LVHR) procedures also needs to be re-assessed [20].

A comparison of data from other studies on mesh surfaces occupied by adhesions, their hardness according to the Zhulke scale and index values is presented in Table III [14, 15]. Polypropylene and polyester meshes in the peritoneal cavity cause the formation of hard adhesions on $\geq 70 \%$ of their surface, which translates into a calculated index $>170$. On the other hand, the index of a 2-layer mesh is 64.6, nearly 3 times lower. Yet a comparison with adhesions formed after classical surgery without mesh application shows that even though the difference in surface area occupied by adhesions in the group without prostheses relative to that with the 2-layer mesh is only $6 \%$, adhesion hardness differs by about 0.7 , which translates into nearly $2 \times$ higher mean index value in the latter group $[13,22,29$, 30]. This difference in index values proves that the mere presence of a mesh prosthesis, in spite of any anti-adhesive properties it may possess, leads to a higher risk of adhesion formation and hardness along with other complications [31, 32].

Comparing these values with other available data on the Dynamesh IPOM mesh and the results of the present study indicates that its susceptibility to adhesion formation is comparable with most commercially available 2-layer meshes. Additionally, the considerable individual variability of index values reported in this study suggests a substantial influence of individual susceptibility on adhesion formation. 
The lack of influence of infections of the fixing suture channel on adhesion hardness in individuals 1,6 , and 8 confirms data from other studies that adhesions of inflammatory etiology have a lower risk of complications relative to those in response to surgical trauma [1].

The second issue relates to the potential influence of fixation elements on adhesion formation. The dependence between types of stabilization systems and visceral adhesion was studied by Hollinsky et al., who showed that non-absorbable ProTack staples significantly increase the risk of adhesion [30]. In the present study, isolated adhesion to fixation sites of fastening sutures was not detected in any of the 24 meshes used (96 points of fastening). This strongly suggests that the slowly absorbable PDSII and Maxon sutures used in this study do not increase the risk of adhesion formation.

In conclusion, the Dynamesh IPOM mesh, in spite of the anti-adhesive layer built of PVDF, does not prevent adhesion formation; the hardness of growing adhesions, their surface area, and index are similar to mean values of these parameters for other similar 2-layer meshes, and slowly absorbable sutures do not increase the risk of adhesion formation.

\section{Acknowledgments}

The authors thank Proper Medical Writing (infrared group s.c.) for technical and language assistance in the preparation of this paper.

\section{References}

1. Brüggmann D, Tchartchian G, Wallwiener M, Münstedt $K$, Tinneberg HR, Hackethal A. Intra-abdominal adhesions definition, origin, significance in surgical practice, and treatment options. Dtsch Arztebl Int 2010; 107: 769-75.

2. Di Vita G, D'Agostino P, Patti R, et al. Acute inflammatory response after inguinal and incisional hernia repair with implantation of polypropylene mesh of different size. Langebecks Arch Surg 2005; 390: 306-11.

3. Heniford BT, Park A, Ramshaw B, Voeller G. Laparoscopic repair of ventral hernias nine years' experience with 850 consecutive hernias. Ann Surg 2003; 238: 391-400.

4. Berger D, Bientzle M, Müller A. Prospective complications after laparoscopic incisional hernia repair. Incidence and treatment. Surg Endosc 2002; 16: 1720-23.

5. Schumpelick V, Klinge U, Welty G, Klosterhalfen B. Meshes within the abdominal wall. Chirurg 1999; 70: 878-87.

6. Adloff M, Arnaud JP. Surgical management of large incisional hernias by an intraperitoneal mesrsilene mesh and an aponeurotic graft. Surg Gynecol Obstet 1987; 165 : 204-6.

7. Kayaoglu HK, Ozkan N, Hazinedaroglu SM, Ersoy OF, Erkek $A B$, Koseoglu RD. Comparison of adhesive properties of five different prosthetic materials used in hernioplasty. J Invest Surg 2005; 18: 89-95.

8. Lo DJ, Bilimoria KY, Pugh CM. Bowel complications after prolene hernia system (PHS) repair: a case report and review of the literature. Hernia 2008; 12: 437-40.
9. Balique JG, Benchetrit S, Bouillot JL, et al. Intraperitoneal treatment of incisional and umbilical hernias using an innovative composite mesh: four-year results of a prospective multicenter clinical trial. Hernia 2005; 9: 68-74.

10. Farrakha M. Laparoscopic treatment of ventral hernia. A bilayer repair. Surg Endosc 2000; 14: 1156-8.

11. Bauer JJ, Harrison MT, Gorfine SR, Kreel I. Rives-Stoppa procedure for repair of large incisional hernias: experience with 57 patients. Hernia 2002; 6: 120-3.

12. Vrijland W, Bonthuis F, Steyerberg W, Marquet RL, Jeekel J, Bonjer HJ. Peritoneal adhesions to prosthetic materials: choice of mesh for incisional hernia repair. Surg Endosc 2000; 14: 960-3.

13. Vavrik J, Foltýnová V, Vítková I, Adámek S, Poučková P. Changes in abdominal wall after mesh implantation in rats. Med Sci Monit 2000; 6: 476-9.

14. Jacob BP, Hogle NJ, Durak E, Kim T, Fowler DL. Tissue ingrowth and bowel adhesion formation in an animal comparative study: polypropylene versus Proceed versus Parietex Composite. Surg Endosc 2007; 21: 629-33.

15. Johanet $\mathrm{H}$, Coelio C. Laparoscopic ventral hernia repair using Parietex Composite mesh: prospective multicenter study. Ann Chir 2004; 129 Spec No 3: 6-8.

16. Schulz DD, Czeczko NG, Malafaia O, et al. Evaluation of healing prosthetic materials polyester mesh reasorbable film and collagen elastin matrix/polypropylene used in rabbits abdominal wall defects. Acta Cir Bras 2009; 24 : 476-83.

17. LeBlanc K. Laparoscopic treatment of ventral hernia. Surg Endosc 2001; 15: 1242.

18. LeBlanc KA, Booth WV, Whitaker JM, Baker D. In vivo study of meshes implanted over the inguinal ring and external iliac vessels in uncastrated pigs. Surg Endosc 1998; 12: 247-51.

19. Weber Gy, Rostas T, Takacs I, et al. Adhesion formation after laparoscopic incisional hernia repair with silicone covered polypropylene mesh a prospective study using abdominal ultrasound. 10th World Congress of Endoscopic Surgery. 2006 Berlin Abstract book 0056.

20. Conze J, Rosch R, Klinge U, et al. Polypropylene in the intra-abdominal position: influence of pore size and surface area. Hernia 2004; 8: 365-72.

21. Takács I, Horváth B, Balatonyi B, et al. Tissue integration of various silicone-coated polypropylene surgical mesh. Magy Seb 2010; 63: 340-6.

22. Klinge $U$, Klosterhalfen $B$, Ottinger $A P$, Junge $K$, Schumpelick V. PVDF as a new polymer for the construction of surgical meshes. Biomaterials 2002; 23: 3487-93.

23. Hollinsky C, Kolbe T, Walter I, et al. Tensile strength and adhesion formation of mesh fixation systems used in laparoscopic incisional hernia repair. Surg Endosc 2010; 24: 1318-24.

24. Ellis H, Moran BJ, Thompson JN, et al. Adhesion-related hospital readmission after abdominal and pelvic surgery: a retrospective cohort study. Lancet 1999; 353: 1476-80.

25. Ott DE. Laparoscopy and adhesion formation, adhesions and laparoscopy. Semin Reprod Med 2008; 26: 322-30.

26. Arnaud JP, Hennekinne-Mucci S, Pessaux P, Tuech JJ, Aube $C$. Ultrasound detection of visceral adhesion after intraperitoneal ventral hernia treatment: a comparative study of protected versus unprotected meshes. Hernia 2003; 7: 85-8.

27. Jonas J. Mesh shrinking and consequences for operation tactics in laparoscopic incisional hernia repair. Hernia 2009; 21 Suppl 1: S33-63. 
28. Śmietanski M, Bigda J, Iwan K, et al. Assessment of usefulness exhibited by different tracks in laparoscopic ventral hernia repair. Surg Endosc 2007; 21: 925-8.

29. Zinther NB, Wara P, Friis-Andersen H. Intraperitoneal onlay mesh: an experimental study of adhesion formation in a sheep model. Hernia 2010; 14: 283-9.

30. Klosterhalfen $\mathrm{B}$, Junge $\mathrm{K}$, Klinge $\mathrm{U}$. The lightweight and large porous mesh concept for hernia repair. Expert Rev Med Devices 2005; 2: 103-17.

31. Vrijland WW, Jeekel J, van Geldorp HJ, Swank DJ, Bonjer HJ. Abdominal adhesions: intestinal obstruction, pain, and infertility Surg Endosc 2003; 17: 1017-22.

32. McGinty JJ, Hogle NJ, McCarthy H, Fowler DL. A comparative study of adhesion formation and abdominal wall ingrowth after laproscopic ventral hernia repair in porcine model using multiple types of mesh. Surg Endosc 2005; 19: 786-90. 